\title{
Magnetic Marking and Intraoperative Detection of Primary Draining Lymph Nodes in High-Risk Prostate Cancer Using Superparamagnetic Iron Oxide Nanoparticles: Additional Diagnostic Value
}

\author{
Alexander Winter ${ }^{*}{ }^{\dagger}$, Svenja Engels ${ }^{\dagger}$, Lena Reinhardt, Clara Wasylow, Holger Gerullis \\ and Friedhelm Wawroschek \\ University Hospital for Urology, Klinikum Oldenburg, School of Medicine and Health Sciences, \\ Carl von Ossietzky University Oldenburg, D-26111 Oldenburg, Germany; \\ engels.svenja@klinikum-oldenburg.de (S.E.); lena@buecken-reinhardt.de (L.R.); claraw98@gmx.de (C.W.); \\ gerullis.holger@klinikum-oldenburg.de (H.G.); wawroschek.friedhelm@klinikum-oldenburg.de (F.W.) \\ * Correspondence: winter.alexander@klinikum-oldenburg.de; Tel.: +49-441-4032302 \\ † These authors contributed equally to this work.
}

Received: 4 November 2017; Accepted: 7 December 2017; Published: 9 December 2017

\begin{abstract}
Sentinel lymph node dissection (sLND) using a magnetometer and superparamagnetic iron oxide nanoparticles (SPIONs) as a tracer was successfully applied in prostate cancer (PCa). Radioisotope-guided sLND combined with extended pelvic LND (ePLND) achieved better node removal, increasing the number of affected nodes or the detection of sentinel lymph nodes outside the established ePLND template. We determined the diagnostic value of additional magnetometer-guided sLND after intraprostatic SPION-injection in high-risk PCa. This retrospective study included 104 high-risk PCa patients (PSA $>20 \mathrm{ng} / \mathrm{mL}$ and/or Gleason score $\geq 8$ and/or cT2c) from a prospective cohort who underwent radical prostatectomy with magnetometer-guided sLND and ePLND. The diagnostic accuracy of sLND was assessed using ePLND as a reference standard. Lymph node metastases were found in 61 of 104 patients (58.7\%). sLND had a 100\% diagnostic rate, $96.6 \%$ sensitivity, $95.6 \%$ specificity, $96.6 \%$ positive predictive value, $95.6 \%$ negative predictive value, $3.4 \%$ false negative rate, and $4.4 \%$ false positive rate (detecting lymph node metastases outside the ePLND template). These findings demonstrate the high sensitivity and additional diagnostic value of magnetometer-guided sLND, exceeding that of ePLND through the individualized extension of PLND or the detection of sentinel lymph nodes/lymph node metastases outside the established node template in high-risk PCa.
\end{abstract}

Keywords: superparamagnetic iron oxide nanoparticles (SPION); prostate cancer; sentinel node; magnetometer; lymphadenectomy

\section{Introduction}

Lymph node (LN) status is a crucial and therapeutically relevant prognostic factor for prostate cancer (PCa). Using LN status, the risks of progression can be calculated and appropriate adjuvant therapy can be planned. There is increasing evidence for the positive therapeutic effects of pelvic LN dissection (PLND) or resecting LN metastases, particularly in patients with minimal LN invasion (LNI) [1-4]. Despite recent advances in imaging, PLND remains the most reliable method for LN staging in clinically localized PCa. However, the reliability of these procedures is limited by their spatial resolution, which limits the sensitivity (49-66\%) of detecting LN (micro)metastases [5]. 
The prevalence of LNI is directly associated with the number of dissected LNs or the extent of PLND [6]. Therefore, the European Association of Urology guidelines recommend an extended PLND (ePLND) approach for LN staging in PCa patients with a $>5 \%$ risk of LNI [7].

Because of the increased complication rate of ePLND and the low detection rate of limited PLND, Wawroschek et al. transferred techniques and concepts of targeted radioisotope-guided sentinel LN (SLN) identification in other tumor entities to PCa [8-10]. In a recent systematic literature review, the diagnostic accuracy of sentinel-guided LN dissection (sLND) was determined by evaluating data from 21 studies (2509 patients). These findings revealed that the diagnostic accuracy of targeted sLND and ePLND were almost the same. sLND combined with ePLND achieved better node removal by increasing the number of affected nodes in $5 \%$ of cases, indicating therapeutic potential [11]. Accordingly, it was demonstrated that sLND yielded higher LNI rates in sentinel cohorts than was expected from established nomograms [12-14]. One reason for this might be the advantage of targeted dissection of tumor-associated LNs or tailoring the extent of PLND to individual lymphatic drainage. Joniau et al. showed that sLND could have an additional diagnostic value over and above ePLND [15]. They showed that $8 \%$ of LN-positive patients would have been missed if only a standard ePLND had been performed.

Nevertheless, due to the ionizing radiation emitted by the technetium-based tracer material, the advantages of the current SLN procedure are accompanied by serious downsides. The dependence on radioisotopes limits the application of this procedure to small parts of the developed world and imposes restrictions on patient planning and hospital logistics. Moreover, the procedure exposes patients and surgical staff to radiation. To overcome these impediments, superparamagnetic iron oxide nanoparticles (SPIONs) have been successfully applied to identify SLNs in breast cancer patients [16,17]. Recently, we presented the first results of intraoperatively detecting SLNs in PCa using a system that comprises a magnetic tracer and a handheld magnetometer [18].

In view of these findings, we hypothesized that magnetometer-guided sLND could have additional diagnostic value because it allows the targeted removal of affected LNs, especially outside the established PLND template in high-risk PCa patients.

To assess the diagnostic accuracy of magnetometer-guided sLND in high-risk PCa, we analyzed high-risk PCa patients from a prospective cohort who underwent radical prostatectomy with magnetometer-guided sLND after intraprostatic SPION injection and ePLND. The diagnostic accuracy of magnetometer-guided sLND was determined using ePLND as a reference standard.

\section{Results}

This study included 104 high-risk PCa patients who underwent radical retropubic prostatectomy with ePLND and magnetometer-guided sLND after intraprostatic injection of SPIONs. Table 1 summarizes the patient characteristics. Median total PSA was $17.21 \mathrm{ng} / \mathrm{mL}$ (interquartile range (IQR) 8.23-32.56).

SLNs were successfully detected by magnetometer-guided sLND in all patients (104/104), resulting in a diagnostic rate of $100 \%$. A total of 845 SLNs were identified. The median number of detected SLNs was 8 (IQR 5-11). SLNs were also localized outside the established ePLND template (e.g., presacral region: 3.3\% and paravesical region: 1.5\%). Figure 1 shows the detailed distribution of all SLNs per anatomic region. 
Table 1. Patient characteristics.

\begin{tabular}{|c|c|c|c|}
\hline & $\begin{array}{l}\text { Overall } \\
n=104\end{array}$ & $\begin{array}{l}\text { Patients with Negative LNs } \\
\quad n=43(41.35 \%)\end{array}$ & $\begin{array}{c}\text { Patients with Positive LNs } \\
\quad n=61(58.65 \%)\end{array}$ \\
\hline Age, years (median) & 69 & 69 & 68 \\
\hline IQR & $63-72.5$ & $63.5-72$ & $62-73$ \\
\hline Total PSA, ng/mL (median) & 17.21 & 12.77 & 21.79 \\
\hline IQR & $8.32-32.56$ & $7.49-24.27$ & $11-41.27$ \\
\hline No. of LNs removed (median) & 17 & 15 & 17 \\
\hline $\mathrm{IQR}$ & $12-21$ & $12-20$ & $13-21$ \\
\hline No. of SLNs removed (median) & 8 & 9 & 7 \\
\hline IQR & $5-11$ & $6-12$ & $5-11$ \\
\hline No. of positive LNs (median) & & & 2 \\
\hline IQR & & & $1-4$ \\
\hline \multicolumn{4}{|l|}{ Tumor stage (\%) } \\
\hline T1c & $23(22.12)$ & $11(25.58)$ & $12(19.67)$ \\
\hline $\mathrm{T} 2 \mathrm{a}$ & $7(6.73)$ & $3(6.98)$ & $4(6.56)$ \\
\hline $\mathrm{T} 2 \mathrm{~b}$ & $6(5.77)$ & $3(6.98)$ & $3(4.92)$ \\
\hline $\mathrm{T} 2 \mathrm{c}$ & $49(47.12)$ & $19(44.19)$ & $30(49.18)$ \\
\hline T3 & $19(18.27)$ & $7(16.28)$ & $12(19.67)$ \\
\hline \multicolumn{4}{|l|}{ Biopsy Gleason score (\%) } \\
\hline $6(3+3)$ & $10(9.62)$ & $8(18.60)$ & $2(3.28)$ \\
\hline $7(3+4)$ & $25(24.04)$ & $11(25.58)$ & $14(22.95)$ \\
\hline $7(4+3)$ & $20(19.23)$ & $7(16.28)$ & $13(21.31)$ \\
\hline$\geq 8$ & $49(47.12)$ & $17(39.53)$ & $32(52.46)$ \\
\hline \multicolumn{4}{|l|}{ Postoperative Gleason score (\%) } \\
\hline $6(3+3)$ & $2(1.94) *$ & $2(4.65)$ & 0 ** \\
\hline $7(3+4)$ & $23(22.33) *$ & $20(46.51)$ & $3(5.00) * *$ \\
\hline $7(4+3)$ & $35(33.98) *$ & $11(25.58)$ & $24(40.00)^{* *}$ \\
\hline$\geq 8$ & $43(41.75)^{*}$ & $10(23.26)$ & $33(55.00) * *$ \\
\hline \multicolumn{4}{|l|}{ Pathologic stage (\%) } \\
\hline pT2 & $28(26.92)$ & $26(60.47)$ & $2(3.28)$ \\
\hline pT3a & $22(21.15)$ & $10(23.26)$ & $12(19.67)$ \\
\hline pT3b & $50(48.08)$ & $7(16.28)$ & $43(70.49)$ \\
\hline pT4 & $4(3.85)$ & 0 & $4(6.56)$ \\
\hline
\end{tabular}

IQR, Interquartile range; (S)LN, (sentinel) lymph node; PSA, prostate specific antigen; */**, data are based on a population of $103\left(^{*}\right)$ and $60\left(^{* *}\right)$ patients (respectively), because one patient underwent hormonal treatment prior to radical retropubic prostatectomy, so there was no postoperative Gleason score.

Taking the ePLND template as a reference standard, sLND results had a $100 \%$ diagnostic rate, 96.6\% sensitivity, $95.6 \%$ specificity, $96.6 \%$ positive predictive value (PPV), $95.6 \%$ negative predictive value (NPV), and a $3.4 \%$ false negative rate.

LN metastases were found in 61 of 104 patients (58.7\%), with a median of two positive LNs (IQR 1-4). Figure 2 shows the distribution of all detected LN metastases per anatomic region.

sLND showed an additional diagnostic value in two of the $61 \mathrm{LN}$-positive patients. In these two cases, sLND detected two LN metastases outside the ePLND template, while ePLND did not reveal any metastases (false positive rate $4.4 \%$ ). The percentage of LN-positive patients with only SLN metastases was $55.7 \%(n=34)$. 


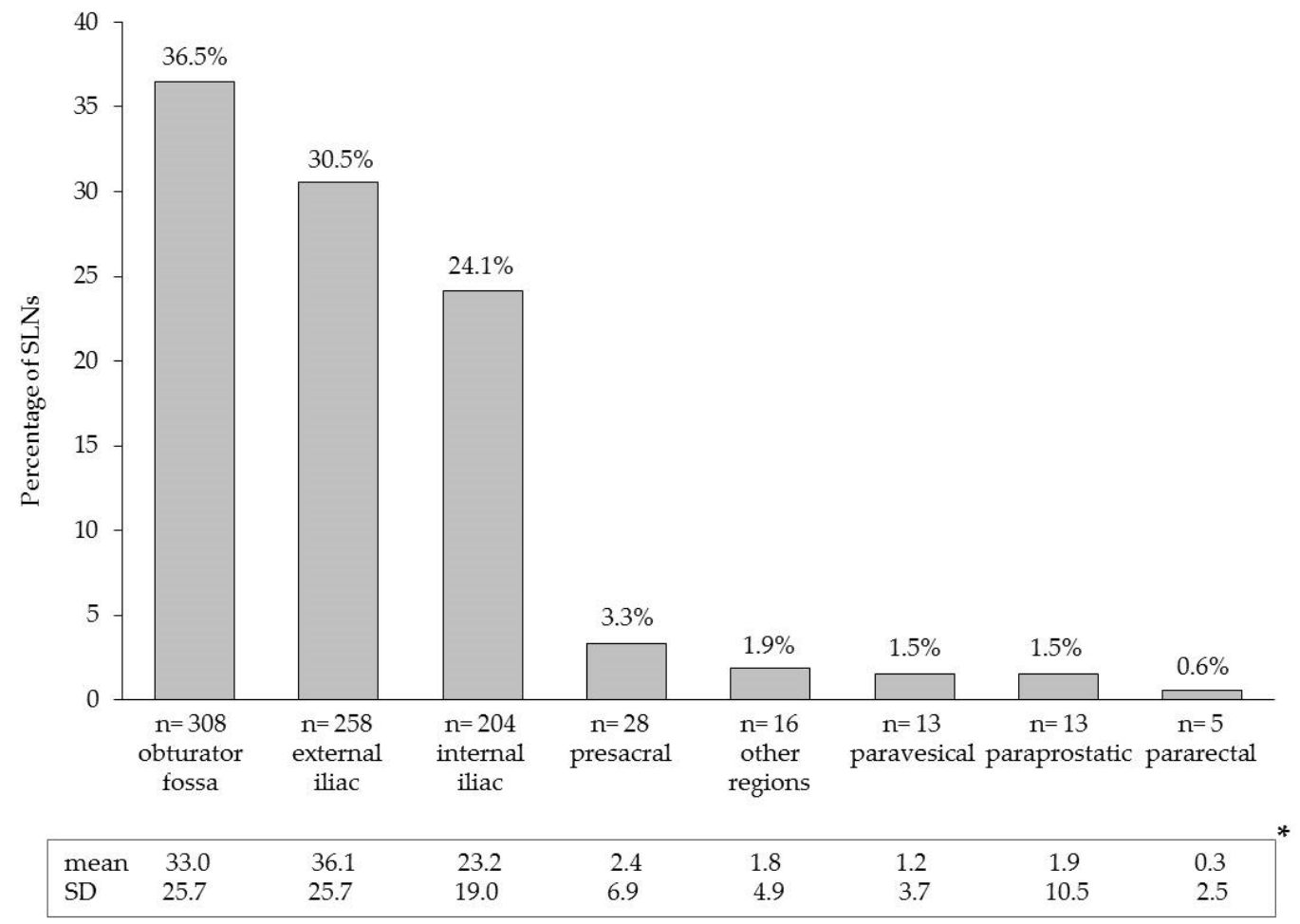

Figure 1. Anatomical distribution of the 845 prostate sentinel lymph nodes from the 104 high-risk patients based on intraoperative magnetometer-guided detection after intraprostatic injection of superparamagnetic iron oxide nanoparticles. * Mean values and standard deviations (SD) on patient level.

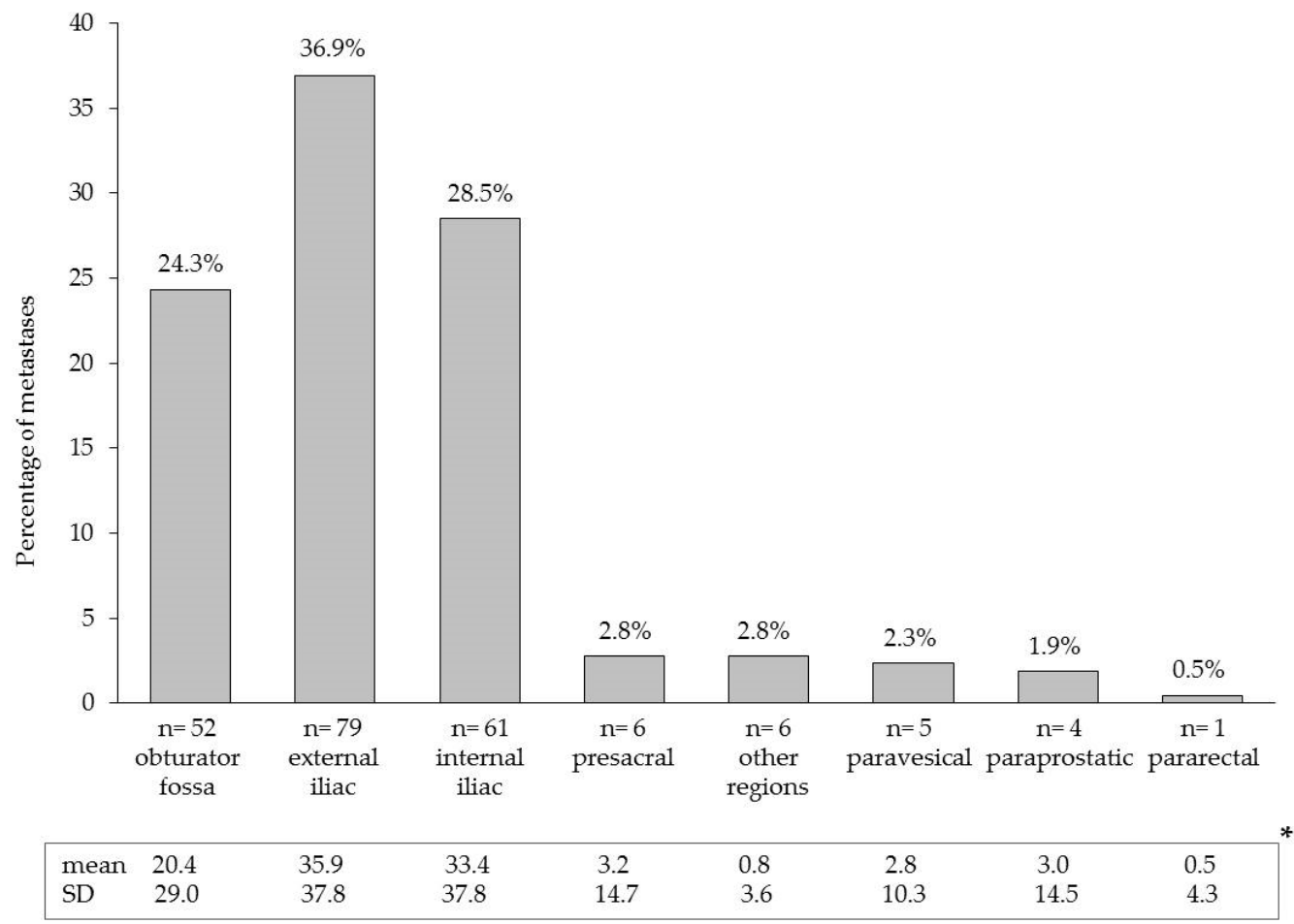

(a)

Figure 2. Cont. 


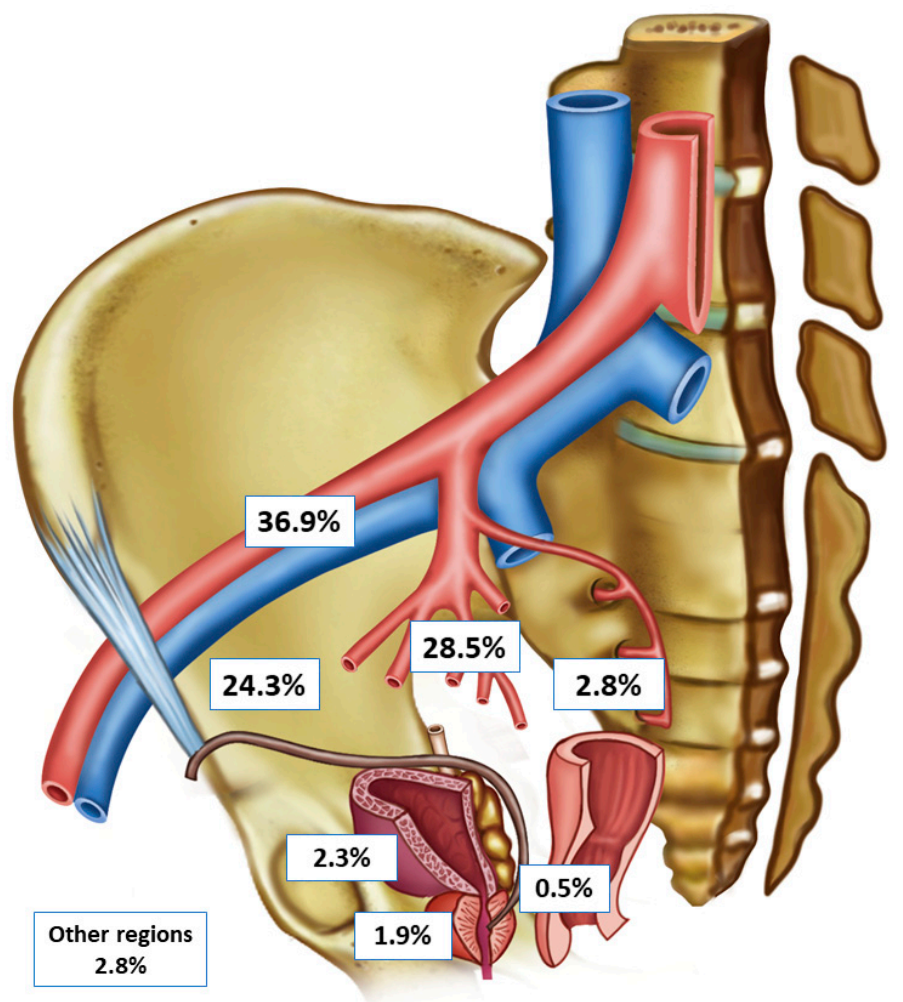

(b)

Figure 2. (a) areas and anatomical distribution of lymph node metastases $(n=214)$ detected by extended pelvic lymph node dissection and/or magnetometer-guided sentinel lymphadenectomy after intraprostatic injection of superparamagnetic iron oxide nanoparticles in 61 lymph node-positive patients with high-risk prostate cancer; (b) distribution and localization of lymph node metastases in an anatomical pelvic model. * Mean values and standard deviations (SD) on a patient level.

\section{Discussion}

The intraoperative use of a handheld magnetometer in combination with SPION-based magnetic resonance imaging after intraprostatic SPION-injection provides a new, entirely radiation-free technique for identifying SLNs in PCa [18,19]. Using this magnetic-targeted sentinel approach, we showed an additional diagnostic value over and above ePLND in high-risk PCa patients. Without the individualized extension of PLND using magnetic SLND, two LN-positive patients ( $3.3 \%$ of all LN-positive patients) would not have been identified.

In this study, which included only high-risk PCa patients, SLNs were intraoperatively identified in all patients. In pilot studies including patients with intermediate-risk and high-risk PCa, we successfully identified SLNs in $89.5 \%$ and $100 \%$ of cases, respectively, using the same magnetic technique $[18,20]$. For radioisotope-guided sLND, Holl et al. showed a detection rate of $98.0 \%$ in a study that included over 2000 low-risk, intermediate-risk, and high-risk PCa patients [21]. A meta-analysis revealed a pooled detection rate of $93.8 \%$ for radio-guided sLND [22]. In a current systematic literature review, the median cumulative percentage for the detection rate was 95.9\% (IQR 89.4-98.5\%) [11]. Accordingly, the magnetic sLND technique works even in high-risk PCa.

However, one fundamental problem of the SLN approach is that when LNs are fully metastasized or when the lymph pathways are blocked, the afferent lymph will be redirected to other LNs/non-SLNs [23]. These LNs cannot be identified using the sentinel procedure, resulting in false-negative findings. The false-negative rate was shown to correlate with Gleason scores [21]; patients with high-risk disease could thus have both positive SLNs and positive non-SLNs or LNI only in non-SLNs. In a study by Weckermann et al., only positive non-SLNs were identified (false-negative 
results) by radioisotope-guided sPLND in two of 96 men with positive LNs (2.1\%) [24]. Therefore, a lower median sensitivity and a higher false-negative rate was observed in studies including only patients at higher risk of LNI [11]. In this study, two of 61 LN-positive patients were not detected by magnetometer-guided sLND, and $44.3 \%$ of LN-positive cases had SLN metastases and additional metastases in non-SLNs.

Conversely, this and other studies have shown an increased detection rate of positive LNs when combining ePLND with sLND or the individualized extension of LN dissection outside the borders of ePLND. For example, Joniau et al. showed that $21 \%$ of preoperatively detected SLNs could be found in the presacral and pararectal region. Moreover, $8 \%$ of LN-positive patients would have been missed if an LN dissection in the presacral region had not been performed [15]. Accordingly, a significant number of SLNs could be visualized outside the standard node template in studies dealing with the radioactive marking approach $[25,26]$. Through the use of magnetic marking, a high proportion of SLNs could be visualized outside the established ePLND template. In total, 24\% of SPION-marked nodes were found, one half each in the presacral and pararectal regions [19]. Results of a current systematic review indicate that one in 20 patients who undergo ePLND, metastatic LNs would have been left behind without performing SLND [11].

As shown in a recent systematic review, there is increasing evidence that surgically removing lymphatic metastases may lead to more favorable outcomes [9]. Thus, there are good arguments for combining sLND with ePLND in high-risk PCa. However, further studies, including a long-term follow-up study, are required to explore the effects of (additional) magnetometer-guided sLND and other SLN techniques on oncologic outcomes.

\section{Materials and Methods}

\subsection{Patients}

This study included 104 consecutive patients with high-risk PCa (European Association of Urology risk group: PSA $>20 \mathrm{ng} / \mathrm{mL}$ and/or Gleason Score $\geq 8$ and/or cT2c [27]) who underwent radical retropubic prostatectomy with magnetometer-guided sLND after intraprostatic injection of SPION and ePLND, which were performed by two highly experienced surgeons at our university center between February 2015 and September 2017. Patients were identified in a prospectively accrued study population. In total, this cohort included 182 patients with intermediate- and high-risk PCa, who had received such intervention. After exclusion of intermediate-risk patients, 104 patients remained. Written informed consent was obtained from all patients.

\subsection{Magnetic SPION Tracer}

The SPION tracer $\left(\right.$ Sienna $\left.+{ }^{\circledR}\right)$ used in these studies is a component of the SentiMag ${ }^{\circledR}$ system (Endomagnetics Ltd., Cambridge, UK). The system for marking and identifying SLNs comprises a handheld magnetometer, the SentiMag ${ }^{\circledR}$ unit, and the Sienna+ ${ }^{\circledR}$ magnet tracer. Both are CE certified as class IIa medical devices. The particles have a carboxydextran coating and a mean hydrodynamic diameter of $60 \mathrm{~nm}$. Sienna+ ${ }^{\circledR}$ has comparable functional properties to that of $99 \mathrm{~m}$ Technetium nanocolloid because, upon interstitial injection, the tracer flows just like the radio nuclide through the lymph system and gets trapped in SLNs.

\subsection{Tracer Injection}

The sentinel technique in PCa differs from those of other tumor types. In breast cancer and malignant melanoma, a well-directed peritumoral injection is only placed to observe the lymphatic drainage of the tumor. In PCa, which commonly occurs as a multifocal malignancy, it is not known with absolute certainty from which part of the organ the metastatic spread originated or which is the index lesion. Therefore, the aim of prostate lymph scintigraphy must be imaging all primary draining LNs of the prostate, under which the SLN of cancer also exists. 
In this study, one urologist injected $2 \mathrm{~mL}$ of SPION (Sienna $+{ }^{\circledR}$ ) into the prostate of patients using transrectal ultrasound guidance $24 \mathrm{~h}$ before surgery. Based on our examinations and those of others, the tracer was evenly spread as three deposits on both sides of the prostate in all cases as described previously [18].

\subsection{Magnetometer-Guided sLND, ePLND, and Histopathological Examination}

Patients underwent magnetometer (SentiMag ${ }^{\circledR}$ )-guided sLND and ePLND, followed by radical retropubic prostatectomy. All cases were performed by two high-volume surgeons, who applied the same anatomic template during ePLND. The ePLND template included the area along the external iliac vessels, with the distal limit being the femoral canal. Proximally, ePLND was carried out to and included the bifurcation of the common iliac artery. All lymphatic fatty tissue along the internal iliac artery and within the obturator fossa and the area dorsal of the obturator nerve was removed, as described by Weingärtner et al. [28]. The lateral limit consisted of the pelvic sidewall, and the medial dissection limit was defined by perivesical fat.

During sLND, all metal retractors were removed from the surgical field, and polymer retractors (SUSI $^{\circledR}$, Aesculap ${ }^{\circledR}$; B. Braun Melsungen AG, Melsungen, Germany) were used to avoid interference with the magnetometer when detecting SLNs with the SentiMag ${ }^{\circledR}$ probe. All SLNs detected by the SentiMag ${ }^{\circledR}$ were removed, whereby each magnetically active LN was seen as an SLN. For surgical reasons, LNs other than SLNs directly adjoining and adhering to SLNs were also removed if in situ separation was not possible. In these cases, LNs were macroscopically detected (tactile and visual) ex vivo and separated by the surgeon from each other or from the containing fibro-fatty tissue. Thereafter, ePLND was conducted to remove remaining lymphatic fatty tissue from the above-named regions. Afterwards, LNs were also macroscopically detected and separated by the surgeon from the containing fibro-fatty tissue.

Postoperatively, all LNs were detected and separated by the surgeon (SLNs and non-SLNs), initially cut in 3-mm transverse sections, routinely processed and embedded in paraffin, while $4-5-\mu \mathrm{m}$-thick sections were stained with hematoxylin-eosin.

\subsection{Outcome Measures of Magnetometer-Guided sLND}

As established by our and other working groups and in line with the results of a recent international sentinel consensus meeting, diagnostic accuracy of sLND was assessed by using conventional ePLND as a reference standard in the same cohort $[11,27,29]$. By complying with this standard, the comparability with the results of other sentinel techniques should be achieved.

The outcomes used to analyze diagnostic test accuracy were diagnostic rate (patients with at least one detected SLN/total number operated), sensitivity, specificity, PPV, NPV, false-positive, and false-negative rates; all were measured at the patient level. False-negative cases were defined as patients with histologically-negative SLN, whilst cancer was found in other LNs. False-positive cases were defined as patients with SLNs containing metastases outside the ePLND template, while the ePLND template did not reveal any metastases [11]. Thus, the false-positive rate provides a measure of the additional diagnostic value of sLND over and above ePLND.

A $2 \times 2$ table with sLND as the index test and ePLND as the reference standard was used to calculate sensitivity, specificity, NPV, and PPV. Additionally, the anatomic distribution of detected LN metastases and identified SLNs were analyzed.

\subsection{Ethical Approval}

All subjects gave their informed consent for inclusion before they participated in the study. The study was conducted in accordance with the Declaration of Helsinki, and the protocol was registered in an international clinical trials register (Research Registry: researchregistry3232). The studies on the prospective cohort were approved by the Ethics Committee of the Medical Chamber 
of Lower Saxony, Germany (no. 24/2014) and the Medical Ethics Committee of the Carl von Ossietzky University Oldenburg (no. 2017-006).

\section{Conclusions}

Magnetometer-guided sLND after intraprostatic injection of SPIONs was successfully applied in PCa. This work demonstrates the high reliability of this new magnetic sentinel approach in detecting LN-positive patients and an additional diagnostic value exceeding that of ePLND in high-risk PCa. Magnetic sLND combined with ePLND achieves better node removal by increasing the number of affected LNs. Increasing evidence of the therapeutic effects of surgically removing LN metastases speaks to the promise of combining sLND with ePLND in high-risk PCa. Further studies, including ones with long-term follow-up, are required to explore the effects of (additional) magnetometer-guided sLND on patient outcomes.

Acknowledgments: Prospective studies on magnetometer-guided sLND were funded by the Research Pool of the Carl von Ossietzky University Oldenburg. The cost to publish as open access was financed by the DFG (Deutsche Forschungsgemeinschaft). We thank James P. Mahaffey, PhD, from Edanz Group (www.edanzediting.com/ac) for editing a draft of this manuscript.

Author Contributions: A.W., S.E., and F.W. conceived and designed the study; A.W. and F.W. performed the surgeries; A.W., F.W., S.E., C.W., and L.R. performed the magnetic measurements; A.W., S.E., C.W., and L.R. analyzed the data; A.W. and S.E. wrote the paper. F.W. and H.G. conducted critical revision for important intellectual content. All authors helped prepare the paper and approved the final version.

Conflicts of Interest: The authors declare no conflict of interest.

\section{Abbreviations}

The following abbreviations are used in this manuscript:

ePLND extended pelvic lymph node dissection

IQR interquartile range

LN lymph node

LNI lymph node invasion

PCa prostate cancer

PLND pelvic lymph node dissection

PSA prostate-specific antigen

SLN sentinel lymph node

sLND sentinel lymph node dissection

SPION superparamagnetic iron oxide nanoparticles

SD standard deviation

\section{References}

1. Choo, M.S.; Kim, M.; Ku, J.H.; Kwak, C.; Kim, H.H.; Jeong, C.W. Extended Versus Standard Pelvic Lymph Node Dissection in Radical Prostatectomy on Oncological and Functional Outcomes: A Systematic Review and Meta-Analysis. Ann. Surg. Oncol. 2017, 24, 2047-2054. [CrossRef] [PubMed]

2. Withrow, D.R.; DeGroot, J.M.; Siemens, D.R.; Groome, P.A. Therapeutic value of lymph node dissection at radical prostatectomy: A population-based case-cohort study. BJU Int. 2011, 108, 209-216. [CrossRef] [PubMed]

3. Winter, A.; Henke, R.P.; Wawroschek, F. Targeted salvage lymphadenectomy in patients treated with radical prostatectomy with biochemical recurrence: Complete biochemical response without adjuvant therapy in patients with low volume lymph node recurrence over a long-term follow-up. BMC Urol. 2015, 15, 10. [CrossRef] [PubMed]

4. Seiler, R.; Studer, U.E.; Tschan, K.; Bader, P.; Burkhard, F.C. Removal of limited nodal disease in patients undergoing radical prostatectomy: Long-term results confirm a chance for cure. J. Urol. 2014, 191, 1280-1285. [CrossRef] [PubMed] 
5. Budäus, L.; Leyh-Bannurah, S.R.; Salomon, G.; Michl, U.; Heinzer, H.; Huland, H.; Graefen, M.; Steuber, T.; Rosenbaum, C. Initial experience of (68)Ga-PSMA PET/CT imaging in high-risk prostate cancer patients prior to radical prostatectomy. Eur. Urol. 2016, 69, 393-396. [CrossRef] [PubMed]

6. Heidenreich, A.; Ohlmann, C.H.; Polyakov, S. Anatomical extent of pelvic lymphadenectomy in patients undergoing radical prostatectomy. Eur. Urol. 2007, 52, 29-37. [CrossRef] [PubMed]

7. Mottet, N.; Bellmunt, J.; Bolla, M.; Briers, E.; Cumberbatch, M.G.; de Santis, M.; Fossati, N.; Gross, T.; Henry, A.M.; Joniau, S.; et al. EAU-ESTRO-SIOG guidelines on prostate cancer. Part 1: Screening, diagnosis, and local treatment with curative intent. Eur. Urol. 2017, 71, 618-629. [CrossRef] [PubMed]

8. Briganti, A.; Chun, F.K.; Salonia, A.; Suardi, N.; Gallina, A.; Da Pozzo, L.F.; Roscigno, M.; Zanni, G.; Valiquette, L.; Rigatti, P.; et al. Complications and other surgical outcomes associated with extended pelvic lymphadenectomy in men with localized prostate cancer. Eur. Urol. 2006, 50, 1006-1013. [CrossRef] [PubMed]

9. Fossati, N.; Willemse, P.M.; van den Broeck, T.; van den Bergh, R.C.N.; Yuan, C.Y.; Briers, E.; Bellmunt, J.; Bolla, M.; Cornford, P.; de Santis, M.; et al. The Benefits and Harms of Different Extents of Lymph Node Dissection during Radical Prostatectomy for Prostate Cancer: A Systematic Review. Eur. Urol. 2017, 72, 84-109. [CrossRef] [PubMed]

10. Wawroschek, F.; Vogt, H.; Weckermann, D.; Wagner, T.; Harzmann, R. The sentinel lymph node concept in prostate cancer-First results of gamma probe-guided sentinel lymph node identification. Eur. Urol. 1999, 36, 595-600. [CrossRef] [PubMed]

11. Wit, E.M.K.; Acar, C.; Grivas, N.; Yuan, C.; Horenblas, S.; Liedberg, F.; Valdes Olmos, R.A.; van Leeuwen, F.W.B.; van den Berg, N.S.; Winter, A.; et al. Sentinel Node Procedure in Prostate Cancer: A Systematic Review to Assess Diagnostic Accuracy. Eur. Urol. 2017, 71, 596-605. [CrossRef] [PubMed]

12. Winter, A.; Kneib, T.; Henke, R.P.; Wawroschek, F. Sentinel lymph node dissection in more than 1200 prostate cancer cases: Rate and prediction of lymph node involvement depending on preoperative tumor characteristics. Int. J. Urol. 2014, 21, 58-63. [CrossRef] [PubMed]

13. Winter, A.; Kneib, T.; Wasylow, C.; Reinhardt, L.; Henke, R.P.; Engels, S.; Gerullis, H.; Wawroschek, F. Updated nomogram incorporating percentage of positive cores to predict probability of lymph node invasion in prostate cancer patients undergoing sentinel lymph node dissection. J. Cancer 2017, 8, 2692-2698. [CrossRef] [PubMed]

14. Grivas, N.; Wit, E.; Tillier, C.; van Muilekom, E.; Pos, F.; Winter, A.; van der Poel, H. Validation and head-to-head comparison of three nomograms predicting probability of lymph node invasion of prostate cancer in patients undergoing extended and/or sentinel lymph node dissection. Eur. J. Nucl. Med. Mol. Imaging 2017. [CrossRef] [PubMed]

15. Joniau, S.; van den Bergh, L.; Lerut, E.; Deroose, C.M.; Haustermans, K.; Oyen, R.; Budiharto, T.; Ameye, F.; Bogaerts, K.; van Poppel, H. Mapping of pelvic lymph node metastases in prostate cancer. Eur. Urol. 2013, 63, 450-458. [CrossRef] [PubMed]

16. Douek, M.; Klaase, J.; Monypenny, I.; Kothari, A.; Zechmeister, K.; Brown, D.; Wyld, L.; Drew, P.; Garmo, H.; Agbaje, O.; et al. Sentinel node biopsy using a magnetic tracer versus standard technique: The SentiMAG Multicentre Trial. Ann. Surg. Oncol. 2014, 21, 1237-1245. [CrossRef] [PubMed]

17. Thill, M.; Kurylcio, A.; Welter, R.; van Haasteren, V.; Grosse, B.; Berclaz, G.; Polkowski, W.; Hauser, N. The Central-European SentiMag study: Sentinel lymph node biopsy with superparamagnetic iron oxide (SPIO) vs. radioisotope. Breast 2014, 23, 175-179. [CrossRef] [PubMed]

18. Winter, A.; Woenkhaus, J.; Wawroschek, F. A novel method for intraoperative sentinel lymph node detection in prostate cancer patients using superparamagnetic iron oxide nanoparticles and a handheld magnetometer: The initial clinical experience. Ann. Surg. Oncol. 2014, 21, 4390-4396. [CrossRef] [PubMed]

19. Winter, A.; Kowald, T.; Paulo, T.; Goos, P.; Engels, S.; Gerullis, H.; Chavan, A.; Wawroschek, F. Magnetic resonance sentinel lymph node imaging in prostate cancer using intraprostatic injection of superparamagnetic iron oxide nanoparticles: The first in-human results. Eur. Urol. Suppl. 2016, 15 (Suppl. 3), 1060. [CrossRef]

20. Winter, A.; Engels, S.; Goos, P.; Süykers, M.C.; Gudenkauf, S.; Henke, R.P.; Gerullis, H.; Wawroschek, F. Magnetometer guided sentinel lymphadenectomy after intraprostatic injection of super-paramagnetic iron oxide nanoparticles in intermediate and high risk prostate cancer patients. J. Urol. 2016, 195 (Suppl. 4), e987. [CrossRef] 
21. Holl, G.; Dorn, R.; Wengenmair, H.; Weckermann, D.; Sciuk, J. Validation of sentinel lymph node dissection in prostate cancer: Experience in more than 2000 patients. Eur. J. Nucl. Med. Mol. Imaging 2009, 36, 1377-1382. [CrossRef] [PubMed]

22. Sadeghi, R.; Tabasi, K.T.; Bazaz, S.M.; Kakhki, V.R.; Massoom, A.F.; Gholami, H.; Zakavi, S.R. Sentinel node mapping in the prostate cancer. Meta-analysis. Nuklearmedizin 2011, 50, 107-115. [CrossRef] [PubMed]

23. Morgan-Parkes, J.H. Metastases: Mechanisms, pathways, and cascades. AJR Am. J. Roentgenol. 1995, 164, 1075-1082. [CrossRef] [PubMed]

24. Weckermann, D.; Dorn, R.; Holl, G.; Wagner, T.; Harzmann, R. Limitations of radioguided surgery in high-risk prostate cancer. Eur. Urol. 2007, 51, 1549-1556. [CrossRef] [PubMed]

25. Mattei, A.; Fuechsel, F.G.; Bhatta Dhar, N.; Warncke, S.H.; Thalmann, G.N.; Krause, T.; Studer, U.E. The template of the primary lymphatic landing sites of the prostate should be revisited: Results of a multimodality mapping study. Eur. Urol. 2008, 53, 118-125. [CrossRef] [PubMed]

26. Wawroschek, F.; Wagner, T.; Hamm, M.; Weckermann, D.; Vogt, H.; Märkl, B.; Gordijn, R.; Harzmann, R. The influence of serial sections, immunohistochemistry, and extension of pelvic lymph node dissection on the lymph node status in clinically localized prostate cancer. Eur. Urol. 2003, 43, 132-136. [CrossRef]

27. Mottet, N.; Bellmunt, J.; Briers, E.; Bolla, M.; Bourke, L.; Cornford, P.; de Santis, M.; Henry, A.M.; Joniau, S.; Lam, T.B.; et al. Guidelines on Prostate Cancer 2017. Available online: http://uroweb.org/guideline/ prostate-cancer/ (accessed on 19 October 2017).

28. Weingärtner, K.; Ramaswamy, A.; Bittinger, A.; Gerharz, E.W.; Vöge, D.; Riedmiller, H. Anatomical basis for pelvic lymphadenectomy in prostate cancer: Results of an autopsy study and implications for the clinic. J. Urol. 1996, 156, 1969-1971. [CrossRef]

29. Van der Poel, H.G.; Wit, E.M.; Acar, C.; van den Berg, N.S.; van Leeuwen, F.W.B.; Valdes Olmos, R.A.; Winter, A.; Wawroschek, F.; Liedberg, F.; Maclennan, S.; et al. Sentinel node prostate cancer consensus panel group members. Sentinel node biopsy for prostate cancer: Report from a consensus panel meeting. BJU Int. 2017, 120, 204-211. [CrossRef] [PubMed]

Sample Availability: Sample Availability: Not available.

(C) 2017 by the authors. Licensee MDPI, Basel, Switzerland. This article is an open access article distributed under the terms and conditions of the Creative Commons Attribution (CC BY) license (http:/ / creativecommons.org/licenses/by/4.0/). 